\title{
Gas-sensitive Field-effect Transistor Incorporating Polymer Layer and Porous Metal Electrode in the Gate Structure
}

\author{
Toshihiro Yoshizumi, Tatsuro Goda, Akira Matsumoto, and Yuji Miyahara* \\ Institute of Biomaterials and Bioengineering, Tokyo Medical and Dental University, \\ 2-3-10 Kanda-Surugadai, Chiyoda, Tokyo 101-0062, Japan
}

(Received November 16, 2017; accepted December 25, 2017)

Keywords: gas-sensitive FET, FET-based sensor, porous gate electrode, stationary phase, volatile organic compound sensing

Field-effect transistor (FET)-based sensors have attractive potential for use in fabricating miniaturized sensor arrays by semiconductor processes. In this work, a gas-sensitive FET incorporating a polyethylene glycol (PEG) film and porous Pt electrode in the gate structure is proposed. In this new type of gas-sensitive FET, the PEG layer provides a dielectric layer (i.e., a stationary phase) that interacts with gaseous molecules, while the porous Pt gate electrode allows gaseous molecules to effectively access the PEG layer. Features of the response pattern, such as peak height, recovery time, and peak shape, unique to the PEG-modified FET sensor were observed when exposed to vapor of volatile organic compounds (VOCs). The sensing mechanism of the gas-sensitive FET is discussed in terms of capacitance changes of the gate structure induced by gaseous molecule adsorption onto the stationary phase. A variety of polymeric materials might be used to modify the gate, and the proposed structure shows promise as a platform for cross-reactive FET-based gas sensor arrays for pattern recognition.

\section{Introduction}

Sensors for gases and vapors of volatile organic compounds (VOCs) have been intensively studied for applications in fields such as disease diagnosis ${ }^{(1-3)}$ and environmental monitoring related to industrial safety and home security. ${ }^{(4)}$ Field-effect transistor (FET)-based sensors have also been widely studied for gas sensing applications. In 1975, Lundström and co-workers first reported a hydrogen-sensitive Pd-gate FET. ${ }^{(5,6)}$ This pioneering work on catalytic gate FETs triggered research into other catalytic gate field-effect devices such as Schottky diode- $(7,8)$ and capacitor-type devices. ${ }^{(9,10)}$ In previous reports, several models were proposed to explain the gas-sensing mechanisms of the catalytic gate field-effect devices. ${ }^{(11,12)}$

The combination of cross-reactive sensing with pattern recognition methods is a promising way to identify gaseous target analytes and estimate their concentrations..$^{(3,13-16)}$ Cross-reactive sensor arrays used for this purpose require broadly responsive sensors with differentiated response profiles. ${ }^{(3)}$ The obtained signals can then be extracted by preprocessing before the

*Corresponding author: e-mail: miyahara.bsr@tmd.ac.jp

http://dx.doi.org/10.18494/SAM.2018.1808 
computational processing. ${ }^{(13-16)}$ FET-based sensors can be downsized and integrated into sensor arrays by standard semiconductor processes, which makes them an ideal platform for crossreactive sensing systems. ${ }^{(17)}$ Furthermore, properties of catalytic gate FETs can be tuned by several methods to achieve broad and differential responses. Several parameters are available for tuning the gas-responsive properties of catalytic gate field-effect devices, including the type of catalytic metal, ${ }^{(11,12)}$ the type of insulating material, ${ }^{(10)}$ and the operation temperature. ${ }^{(12)}$

Here, we describe a new approach to fine-tuning the gas-responsive properties of FETs in an approach compatible with the cross-reactive sensor array applications [Fig. 1 (a)]. The gas-sensitive FET we propose incorporates a structure based on a porous Pt gate electrode embedded into a gas-adsorbing layer, i.e., polyethylene glycol (PEG). The FET operates in the $n$-channel depletion mode. As shown in Fig. 1(a), the insulating layers are composed of $\mathrm{SiO}_{2}$, $\mathrm{Si}_{3} \mathrm{~N}_{4}$, and $\mathrm{Ta}_{2} \mathrm{O}_{5}$; note that the presence of the $\mathrm{Ta}_{2} \mathrm{O}_{5}$ layer is not an absolute requirement for the present proof-of-concept study. PEG, which is widely used as a stationary phase in capillary columns for gas chromatography $(\mathrm{GC}){ }^{(18)}$ is used as a gas-adsorbing layer in the gate structure. Furthermore, a porous Pt layer is formed as the gate electrode directly on the PEG layer, which thus allows gaseous analytes to access the PEG layer.

\section{Materials and Methods}

To develop the new gas-sensitive FET combined with a PEG layer and porous Pt gate electrode, we used a commercially available base FET without a gate electrode. To form a PEG layer, $2 \mu \mathrm{L}$ of PEG (PEG 20M, Shinwa Chemical Industries) -dissolved Milli-Q pure water $\left(0.3 \mathrm{~g} \mathrm{~L}^{-1}\right)$ was dropped onto the surface of the $\mathrm{Ta}_{2} \mathrm{O}_{5}$ layer and the solution was evaporated in an oven at $110{ }^{\circ} \mathrm{C}$ for $1 \mathrm{~h}$. After the formation of a PEG layer, a porous Pt gate electrode was deposited by RF sputtering at a power of $50 \mathrm{~W}$ for 30 or $60 \mathrm{~s}$ (Table 1) in a vacuum chamber (Ar atmosphere of $\sim 1 \mathrm{~Pa}$ ). In the characterization of the new gate structure, the 1-min-sputtered FET (device 1) was observed with a scanning electron microscope (SEM; JSM-7500F, JEOL), transmission electron microscope (TEM; JEM-2100F, JEOL), and scanning TEM (STEM; JEM2100F, JEOL). To obtain cross sections of the gate structure, the FET device was protected

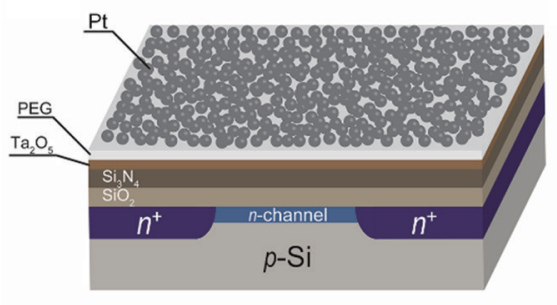

(a)

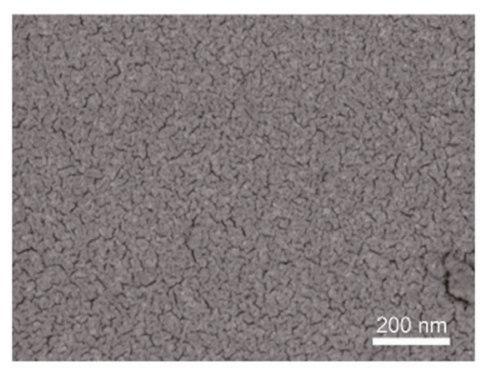

(b)

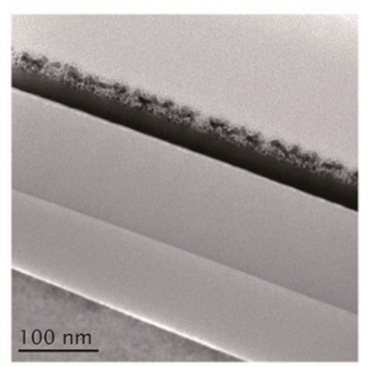

(c)

Fig. 1. (Color online) (a) Schematic illustration of FET incorporating PEG layer and porous Pt gate electrode. The FET operates in the n-channel depletion mode. (b) SEM image of the surface of a porous Pt gate electrode. Scale bar is $200 \mathrm{~nm}$. (c) TEM images of cross section of the gate structure. Scale bar is $100 \mathrm{~nm}$. 
Table 1

PEG layer and sputtering time details of device.

\begin{tabular}{lcc}
\hline Device No. & PEG Layer & Sputtering time \\
\hline 1 & Formed & $1 \mathrm{~min}$ \\
2 & Formed & $30 \mathrm{~s}$ \\
3 & None & $30 \mathrm{~s}$ \\
\hline
\end{tabular}

with resin and then processed with a focused ion beam system (JIB-4501, JEOL). Elemental mapping of the cross section was performed in the STEM mode combined with energydispersive X-ray spectroscopy (EDX; JED-2300T, JEOL). The vapor samples were generated with a vapor-generator apparatus (Permeater PD-1B-2, GASTEC) and introduced into the gas-flow cell containing the developed FET sensor with dry $\mathrm{N}_{2}$ carrier gas. Dry $\mathrm{N}_{2}$ carrier gas was selected as the inert atmospheric gas for avoiding the effect of moisture. During measurements of the electronic properties, we heated the FET device to $70{ }^{\circ} \mathrm{C}$ on a hot plate and the temperature of the device was measured with a thermocouple. Drain current $\left(I_{D}\right)$-gate voltage $\left(V_{G}\right)$ curves of device 1 were measured using a semiconductor parameter analyzer (4155C, Agilent Technologies) at a drain-source voltage $\left(V_{D S}\right)$ of $1 \mathrm{~V}$. Real-time response measurements of the FET devices to vapor samples were conducted with a measuring instrument based on the electronic circuit shown in Fig. 2. In the electronic circuit, changes in output voltage $\left(\Delta V_{\text {out }}\right)$ occur alongside a shift in the threshold voltage $\left(\Delta V_{T}\right)$ of the device. During response measurements, $V_{G}$ and $V_{D S}$ were fixed at 0.2 and $4 \mathrm{~V}$, respectively.

\section{Results and Discussion}

The SEM image in Fig. 1(b) shows a top view of the surface of the Pt gate electrode. A markedly porous morphology consisting of discontinuous Pt is apparent in Fig. 1(b). Figure. 3 shows STEM images and EDX elemental map of N, O, Si, Ta, and Pt. The elemental mapping revealed that the gate structure was composed of multiple layers in the desired arrangement. It was confirmed that the porous Pt gate electrode occupied the top layer. In carbon mapping, unfortunately, the PEG layer could not be distinguished from the resin layer used to support the structure in the cutting process. Cross-sectional TEM images shown in Fig. 1(c) indicate that the porous morphology of the Pt electrode consisted of a network of Pt nanoparticles (NPs), including some Pt NP structures that partially infiltrated the PEG layer.

Representative $I_{D}-V_{G}$ curves of device 1 upon exposure to ethanol vapor (38 ppm) are shown in Fig. 4. The introduction of ethanol vapor produced a negative shift of $V_{T}$ in the FET device. A plausible explanation for this behavior may be given by taking into account the change in the overall capacitance of the gate environment as follows.

The working principle of a FET in the unsaturated region can be generally expressed as ${ }^{(19,20)}$

$$
I_{D}=\mu C_{O X} \frac{W}{L}\left[\left(V_{G}-V_{T}\right) V_{D S}-\frac{1}{2} V_{D S}^{2}\right],
$$




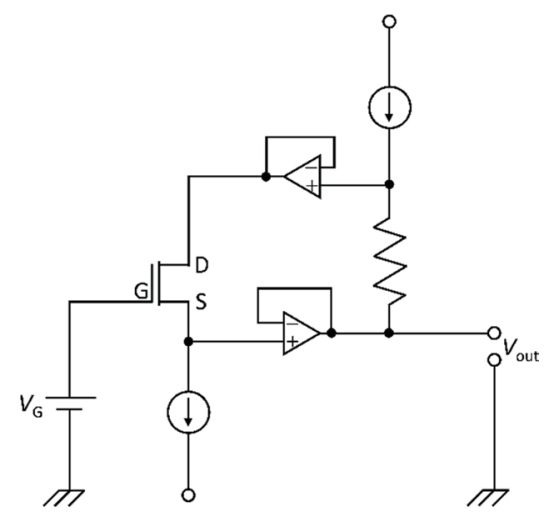

Fig. 2. Electronic circuit used for real-time measurements of gas response characteristics of the FET.

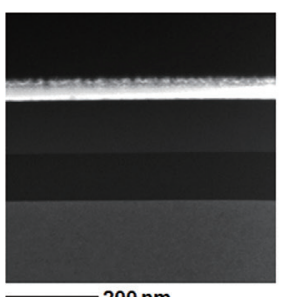

(a)

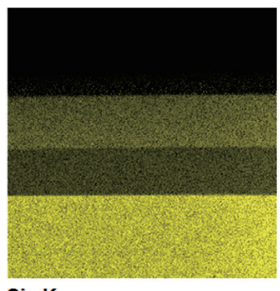

(d)

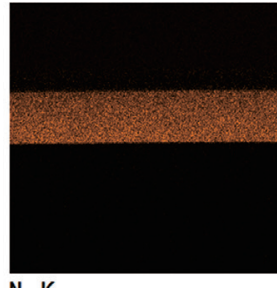

(b)

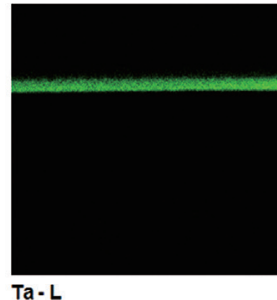

(e)

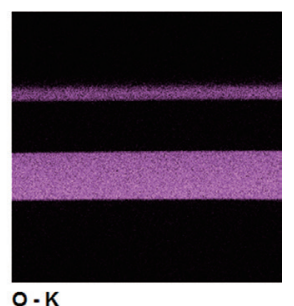

(c)

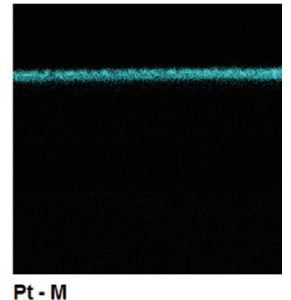

(f)

Fig. 3. (Color online) (a) STEM image and (b)-(f) elemental mapping of cross section of the FET (device 1) by STEM-EDX: (b) Nitrogen, (c) oxygen, (d) silicon, (e) tantalum, and (f) platinum mapping. Scale bar is $200 \mathrm{~nm}$.

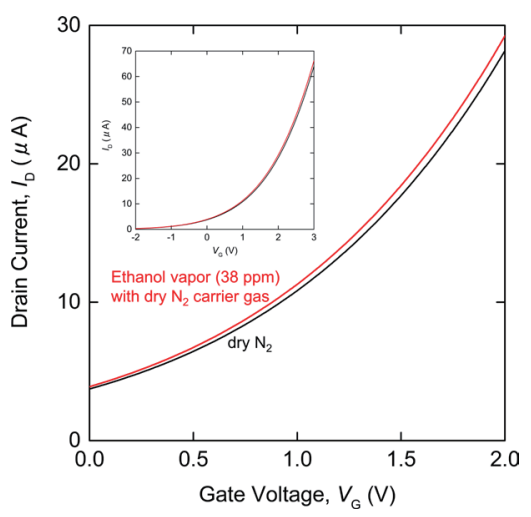

Fig. 4. (Color online) $I_{D}-V_{G}$ curves $\left(V_{D S}: 1 \mathrm{~V}\right)$ of the FET of device 1 when ethanol vapor with dry $\mathrm{N}_{2}$ carrier gas was introduced into the gas-flow cell container. (Red) $38 \mathrm{ppm}$ ethanol vapor with dry $\mathrm{N}_{2}$ and (black) only dry $\mathrm{N}_{2}$. 
where $I_{D}$ is the drain current, $\mu$ is the electron mobility in the channel, $C_{O X}$ is the gate oxide capacitance per unit area, and $\frac{W}{L}$ is the channel width-to-length ratio. $V_{G}, V_{T}$, and $V_{D S}$ are the applied gate, threshold, and drain-source voltages, respectively. Furthermore, $V_{T}$ can be expressed as

$$
V_{T}=V_{F B}-\frac{Q_{B}}{C_{O X}}+2 \varphi_{f}
$$

where $V_{F B}$ is the flat band voltage, $Q_{B}$ is the bulk depletion charge per unit area, and $\varphi_{f}$ is the Fermi potential difference between the doped bulk silicon and intrinsic silicon.

Here, we assume that the intrinsic dipole moment of a polar molecule adsorbed onto a polymer can generate changes in the overall capacitance value of the stationary phase. ${ }^{(20)}$ For a polymer-modified FET, Eqs. (1) and (2) should be modified as

$$
\begin{gathered}
I_{D}=\mu C_{c o m} \frac{W}{L}\left[\left(V_{G}-V_{T}\right) V_{D S}-\frac{1}{2} V_{D S}^{2}\right], \\
V_{T}=V_{F B}-\frac{Q_{B}}{C_{c o m}}+2 \varphi_{f},
\end{gathered}
$$

with

$$
\begin{gathered}
C_{c o m}=\frac{C_{i n} \times C_{s t}}{C_{i n}+C_{s t}}=\frac{C_{s t}}{1+\frac{C_{s t}}{C_{i n}}}, \\
C_{s t}=\varepsilon_{0} \varepsilon_{r} \frac{S}{d},
\end{gathered}
$$

where $C_{i n}$ and $C_{s t}$ are the capacitances of the insulating layer and the stationary phase (polymer layer), respectively. $C_{c o m}$ is the sum capacitance of $C_{i n}$ and $C_{s t}$. The parameters $\varepsilon_{0}, \varepsilon_{r}, S$, and $d$ are the vacuum permittivity, relative static permittivity, area, and thickness of the polymer layer, respectively.

Adsorption of polar molecules onto the stationary phase induces a change in the permittivity in the stationary phase, resulting in a change in $C_{s t}\left(\Delta C_{s t}\right)$. As expressed in Eq. (5), this result qualitatively demonstrates that $\Delta C_{s t}$ can generate a change in $C_{c o m}\left(\Delta C_{c o m}\right)$, that affects $V_{T}$ and $I_{D}$ of the FET.

The thickness and density of the Pt layer depend on sputtering time and can affect the sensitivity of the FET-based sensor. For example, it has been reported that a smaller thickness of the Pt gate electrode provides higher sensitivity in a solid-electrolyte-based FET oxygen sensor. ${ }^{(21)}$ Figure 5(a) shows the real-time response of the developed FET sensors, 30 -s-sputtered devices (devices 2 and 3), when exposed to hexanal vapor samples of various concentrations. The red-highlighted regions represent intervals during which the vapor samples 


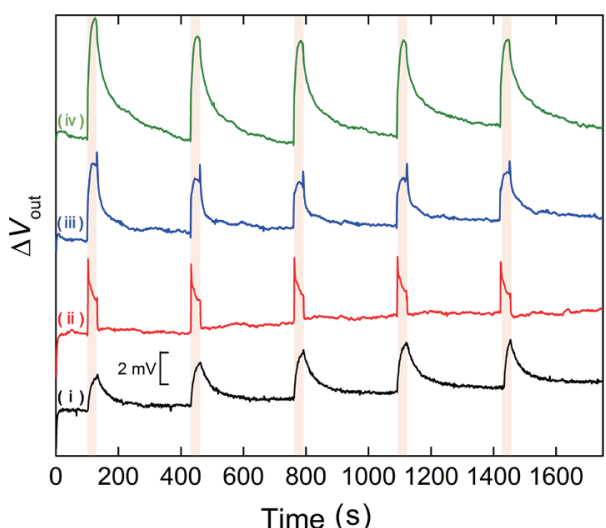

(a)

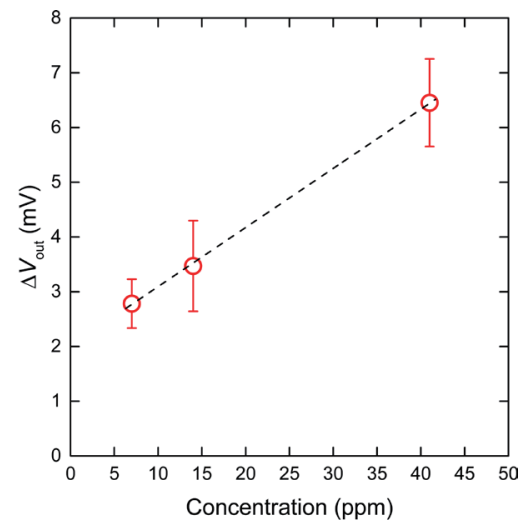

(b)

Fig. 5. (Color online) (a) Real-time measurements of the response of FET devices to hexanal vapor. (i) Device 3 and (ii-iv) device 2. (i, ii) $7 \mathrm{ppm}$. (iii) $14 \mathrm{ppm}$. (iv) $41 \mathrm{ppm}$. (b) Hexanal vapor concentration dependence of $\Delta V_{\text {out }}$ in device 2. Dashed line is a guide for the eyes.

were introduced. In the principle of the electronic circuit shown in Fig. 2, the rise of $V_{\text {out }}$ induced by VOC exposure indicates a negative shift of $V_{T}$. As evident from Fig. 5(a), the $\Delta V_{\text {out }}$ responses to hexanal vapor were reversible and could be cycled multiple times. A comparison between the two devices with (device 2) and without (device 3) the PEG layer suggested that the PEG layer contributed to considerable differences in the response patterns, including the peak height, recovery time, and peak shape. The relationship between the hexanal vapor concentration and $\Delta V_{\text {out }}$ of device 2 is shown in Fig. 5(b). The value of $\Delta V_{\text {out }}$ increased as the hexanal concentration increased. Furthermore, the recovery time of $\Delta V_{\text {out }}$ to the baseline also increased as the hexanal concentration increased, as observed in Fig. 5(a). These results can be attributed to greater amounts of vapor adsorbing on the PEG layer and Pt NPs. The increased adsorption of gaseous molecules led to an increase in $\Delta V_{\text {out }}$ and a longer recovery time for desorption. To evaluate the repeatability in Fig. 5(a), we calculated the coefficient of variation $(\mathrm{CV})$ of $\Delta V_{\text {out }}$ between the baseline and peak using the standard deviation and mean value. The CV values in measurements of 7, 14, and $41 \mathrm{ppm}$ of hexanal vapor were 16,24 , and $12 \%$, respectively.

To demonstrate the response of the developed gas-sensitive FET to a broad range of gaseous analytes, we exposed device 2 to several VOC samples and monitored $\Delta V_{\text {out }}$. Figure 6 shows real-time measurements of the response characteristics of the gas-sensitive FET (device 2) to vapors of ethanol (17 ppm), 1-propanol (10 ppm), tetrahydrofuran (42 ppm), acetone (64 ppm), and nonanal $(2 \mathrm{ppm})$. Figure 6 reveals that the $\Delta V_{\text {out }}$ signals were reproducible with repeated exposure to the VOCs. Furthermore, the FET-based sensor was broadly responsive toward these VOCs. As mentioned in the introduction, these broadly responsive properties could be useful for the fabrication of cross-reactive sensor arrays. 


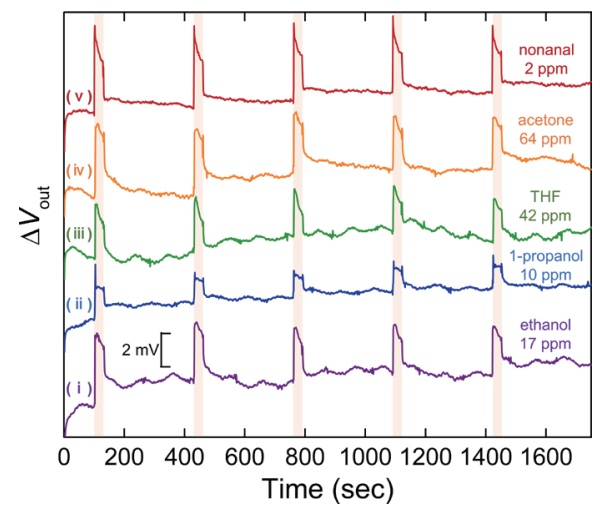

Fig. 6. (Color online) Real-time measurements of response of device 2 to vapor. (i) Ethanol: $17 \mathrm{ppm}$. (ii) 1-Propanol: 10 ppm. (iii) Tetrahydrofuran: 42 ppm. (iv) Acetone: 64 ppm. (v) Nonanal: 2 ppm.

\section{Conclusions}

Here, we reported a new gas-sensitive FET incorporating a polymer film with a porous electrode in the gate structure. PEG, which is widely used as a stationary phase material for GC, was applied to the gate structure to provide adsorption sites for gaseous molecules. Furthermore, a porous Pt layer that allows gaseous molecules to access the PEG layer was used as the gate electrode. When exposed to vapor of VOCs, the responsive patterns were unique to the PEG-modified FET in terms of peak height, recovery time, and peak shape. In addition, the proposed FET showed a broad response toward VOC vapors. A variety of different types of polymers can be applied to tailor adsorption properties and, thus, the profile of response. Although the response mechanism has yet to be clarified in detail, the FET structure described herein could be useful as a platform for cross-reactive sensor arrays coupled with pattern recognition methods, which require individual sensing components that can detect a broad range of analytes with differential responses.

\section{Acknowledgments}

This work was supported by the ImPACT Program of the Council for Science, Technology and Innovation.

\section{References}

1 A. P. F. Turner and N. Magan: Nat. Rev. Microbiol. 2 (2004) 161.

2 G. Konvalina and H. Haick: Acc. Chem. Res. 47 (2014) 66.

3 R. Vishinkin and H. Haick: Small 11 (2015) 6142.

4 R. A. Potyrailo: Chem. Rev. 116 (2016) 11877.

5 I. Lundström, S. Shivaraman, C. Svensson, and L. Lundkvist: Appl. Phys. Lett. 26 (1975) 55.

6 I. Lundström, M. S. Shivaraman, and C. M. Svensson: J. Appl. Phys. 46 (1975) 3876. 
7 M. C. Steele and B. A. Maclver: Appl. Phys. Lett. 28 (1976) 687.

8 K. Ito: Surf. Sci. 86 (1979) 345.

9 F. Winquist, A. Spetz, M. Armgarth, C. Nylander, and I. Lundström: Appl. Phys. Lett. 43 (1983) 839.

10 M. Eriksson, A. Salomonsson, I. Lundström, D. Briand, and A. E. Åbom: J. Appl. Phys. 98 (2005) 034903.

11 I. Lundström, H. Sundgren, F. Winquist, M. Eriksson, C. Krantz-Rülcker, and A. Lloyd-Spetz: Sens. Actuators, B 121 (2007) 247.

12 I. Lundström, A. Spetz, F. Winquist, U. Ackelid, and H. Sundgren: Sens. Actuators, B 1 (1990) 15.

13 W. Göpel: Sens. Actuators, B 52 (1998) 125.

14 K. J. Albert, N. S. Lewis, C. L. Schauer, G. A. Sotzing, S. E. Stitzel, T. P. Vaid, and D. R. Walt: Chem. Rev. $100(2000) 2595$.

15 P. C. Jurs, G. A. Bakken, and H. E. McClelland: Chem. Rev. 100 (2000) 2649.

16 A. Hierlemann and R. Gutierrez-Osuna: Chem. Rev. 108 (2008) 563.

17 H. Sundgren, I. Lundström, and F. Winquist: Sens. Actuators, B 2 (1990) 115.

18 E. F. Barry and R. L. Grob: Columns for Gas Chromatography: Performance and Selection (Wiley, Hoboken, 2007) Chap. 3

19 P. Bergveld: Sens. Actuators 8 (1985) 109.

20 A. Matsumoto, N. Sato, T. Sakata, R. Yoshida, K. Kataoka, and Y. Miyahara: Adv. Mater. 21 (2009) 4372.

21 Y. Miyahara, K. Tsukada, and H. Miyagi: J. Appl. Phys. 63 (1988) 2431. 